01;03;13

\title{
Ток насыщения стационарного конического образования на поверхности жидкости с ионной проводимостью в электрическом поле
}

\author{
(C) М.А. Беляев ${ }^{1}$, Н.М. Зубарев ${ }^{1,2, \uparrow, ~ О . В . ~ З у б а р е в а ~}{ }^{1}$ \\ ${ }^{1}$ Институт электрофизики УрО РАН, Екатеринбург, Россия \\ ${ }^{2}$ Физический институт им. П.Н. Лебедева РАН, Москва, Россия \\ ฯ E-mail: nick@iep.uran.ru
}

Поступило в Редакцию 28 января 2019 г.

В окончательной редакции 28 января 2019 г.

Принято к публикации 1 февраля 2019 г.

Исследуются конические структуры, возникающие на поверхности жидкости с ионной проводимостью в электрическом поле, с учетом влияния объемного и поверхностного зарядов. Демонстрируется, что задача о распределении поля допускает точное аналитическое решение, соответствующее режиму ограничения тока электрическим зарядом. Найдена зависимость тока насыщения через коническое образование от угла раствора, диэлектрической проницаемости жидкости и подвижности положительных и отрицательных ионов.

DOI: 10.21883/PJTF.2019.08.47617.17714

Известно, что при воздействии достаточно сильного электрического поля свободная поверхность жидкости становится неустойчивой [1-7]. В результате на ее поверхности могут формироваться стационарные конические структуры [8-10]. Впервые такие структуры были теоретически описаны Тейлором [11] для случая идеально проводящей жидкости. Было установлено, что угол полураствора образующихся конусов составляет $\beta_{T} \approx 49.3^{\circ}$. Позднее этот результат был обобщен на случай идеальной диэлектрической жидкости [12] (см. [13] для магнитной жидкости в магнитном поле). Как оказалось, конические образования могут возникать на поверхности жидкостей с достаточно большой диэлектрической проницаемостью $(\varepsilon>17.6)$. Угол полураствора конуса $\beta$ при этом зависит от $\varepsilon$ и попадает в интервал $0<\beta<\beta_{T}$. Автомодельные решения уравнений электрогидродинамики, описывающие зарождение конусов, были найдены в $[14,15]$.

В недавних теоретических работах $[16,17]$ было рассмотрено протекание тока через коническое образование на поверхности жидкости при наличии поверхностного и объемного заряда ионов различного знака (допускалась генерация ионов в области сильного поля у вершины конуса). Было дано физическое обоснование использования приближения, в котором поверхностный заряд положительных ионов полностью экранирует нормальную компоненту напряженности поля в жидкости, а в объеме жидкого конуса доминируют отрицательные ионы. Анализ проводился для конуса с предельно малым углом раствора $(\beta / \pi \ll 1)$ для жидкости со значительной проницаемостью $(\varepsilon \gg 1)$.

В настоящей работе, следуя $[16,17]$, мы рассматриваем коническое образование на поверхности жидкости с ионной проводимостью в направленном от жидкости внешнем электрическом поле. По поверхности конуса в направлении его вершины дрейфуют положительные ионы (катионы), а от вершины конуса по его объему дрейфуют отрицательные ионы (анионы). Как показано далее, задача нахождения распределения электрического поля для такой системы допускает построение точного аналитического решения без ограничений на диэлектрическую проницаемость $\varepsilon$ и угол раствора конуса $\beta$. Решение соответствует режиму ограничения тока электрическим зарядом и, как следствие, позволяет определить максимально возможные значения тока, протекающего через коническое образование, вне зависимости от механизма генерации ионов.

Выпишем основные уравнения. Будем считать, что в области вне жидкого конуса отсутствует существенный объемный электрический заряд и, следовательно, распределение потенциала электрического поля удовлетворяет уравнению Лапласа $\Delta \Phi_{1}=0$ (здесь и далее индексы 1 и 2 относятся к областям вне и внутри жидкости соответственно). В дальнейшем будем использовать сферическую систему координат, т.е. примем $\Phi_{1}=\Phi_{1}(R, \theta)$, где $R$ - расстояние от вершины конуса, a $\theta$ - полярный угол. В ней уравнение Лапласа примет вид

$$
\frac{\partial^{2} \Phi_{1}}{\partial R^{2}}+\frac{2}{R} \frac{\partial \Phi_{1}}{\partial R}+\frac{1}{R^{2}} \frac{\partial^{2} \Phi_{1}}{\partial \theta^{2}}+\frac{\operatorname{ctg} \theta}{R^{2}} \frac{\partial \Phi_{1}}{\partial \theta}=0 .
$$

Внутри жидкого конуса имеется нескомпенсированный электрический заряд, обусловленный дрейфом анионов. Распределение электрического поля в этой области описывается уравнением Пуассона: $\Delta \Phi_{2}=-\rho /\left(\varepsilon_{0} \varepsilon\right)$, где $\rho$ - объемная плотность заряда, а $\varepsilon_{0}$ - электрическая постоянная. Следуя $[16,17]$, будем считать, что на границе жидкого конуса (она задается условием $\theta=\Theta \equiv \pi-\beta)$ имеется свободный положительный поверхностный заряд плотностью $\sigma$, который полностью 
экранирует нормальную компоненту электрического поля внутри жидкости (отметим, что в этом случае поляризационный заряд на границе не индуцируется), т.е.

$$
\frac{\partial \Phi_{2}}{\partial \theta}=0, \quad \theta=\Theta .
$$

Тогда можно предположить, что в жидкости имеется только радиальная компонента электрического поля, т.е. $\Phi_{2}=\Phi_{2}(R)$ и $\rho=\rho(R)$. В этом случае уравнение Пуассона запишется как

$$
\frac{\partial^{2} \Phi_{2}}{\partial R^{2}}+\frac{2}{R} \frac{\partial \Phi_{2}}{\partial R}=-\frac{\rho}{\varepsilon_{0} \varepsilon} .
$$

Плотность зарядов $\rho$ должна удовлетворять стационарному уравнению непрерывности $\nabla\left(\rho \mathbf{V}_{a}\right)=0$, которое с учетом выражения $\mathbf{V}_{a}=\mu_{a} \nabla \Phi_{2}$ для скорости дрейфа анионов $\left(\mu_{a}-\right.$ их подвижность $)$ примет следующий вид:

$$
\rho\left(\frac{\partial^{2} \Phi_{2}}{\partial R^{2}}+\frac{2}{R} \frac{\partial \Phi_{2}}{\partial R}\right)+\frac{\partial \rho}{\partial R} \frac{\partial \Phi_{2}}{\partial R}=0 .
$$

По поверхности конуса течет ток, переносимый катионами. Скорость катионов в электрическом поле определяется их подвижностью: $\mathbf{V}_{c}=-\mu_{c} \nabla \Phi_{2}$. Уравнение непрерывности для катионов с учетом этой связи запишется как

$$
\sigma\left(\frac{\partial^{2} \Phi_{2}}{\partial R^{2}}+\frac{1}{R} \frac{\partial \Phi_{2}}{\partial R}\right)+\frac{\partial \sigma}{\partial R} \frac{\partial \Phi_{2}}{\partial R}=0 .
$$

Приведем необходимые граничные условия. Тангенциальные компоненты электрического поля внутри и вне жидкости будут совпадать на ее границе, а нормальная компонента поля должна испытывать разрыв, пропорциональный плотности заряда на поверхности конуса:

$$
\frac{\partial \Phi_{1}}{\partial R}=\frac{\partial \Phi_{2}}{\partial R}, \quad \frac{1}{R} \frac{\partial \Phi_{1}}{\partial \theta}=\frac{\sigma}{\varepsilon_{0}}, \quad \theta=\Theta
$$

где мы учли дополнительное условие (2). Также должно выполняться условие симметрии для потенциала поля

$$
\frac{\partial \Phi_{1}}{\partial \theta}=0, \quad \theta=0 .
$$

Наконец, условием равновесия свободной поверхности жидкости является равенство на ней электростатического и капиллярного давлений. Для конуса с углом раствора $2 \beta$ это дает

$$
\begin{gathered}
p_{E}=p_{S}, \\
p_{E}=\frac{\varepsilon_{0}}{2}\left(\frac{1}{R^{2}}\left(\frac{\partial \Phi_{1}}{\partial \theta}\right)^{2}+(\varepsilon-1)\left(\frac{\partial \Phi_{1}}{\partial R}\right)^{2}\right)_{\theta=\Theta}, \\
p_{S}=-\frac{\alpha}{R} \operatorname{ctg} \Theta,
\end{gathered}
$$

где $\alpha-$ коэффициент поверхностного натяжения жидкости.
Из условия баланса давлений (8) следует, что $p_{E} \propto R^{-1}$. Тогда по аналогии с классическим результатом [11] следует ожидать, что напряженность поля будет убывать с удалением от вершины конуса как $R^{-1 / 2}$, а потенциал поля соответственно удовлетворять скейлингу $\Phi \propto R^{1 / 2}$. Как видно из (3), для плотности заряда должно выполняться $\rho \propto R^{-3 / 2}$, что согласуется с уравнением (4). Далее из (6) следует, что для плотности поверхностного заряда должен реализовываться скейлинг $\sigma \propto R^{-1 / 2}$, который в свою очередь согласуется с (5). То обстоятельство, что учет объемного заряда дрейфующих в конической области носителей тока не нарушает классического скейлинга $\Phi \propto R^{1 / 2}$ (т. е. возможно согласованное решение уравнений типа (3), (4) и (8)), было, по-видимому, впервые установлено в работе [18] для потока заряженных капель, эмитируемых с вершины конуса Тейлора. То, что учет поверхностного тока также не нарушает подобного скейлинга, т.е. возможно построение согласованного решения уравнений $(5),(6)$ и (8), было показано в [19].

Итак, рассматриваемая задача, требующая учета влияния как объемного, так и поверхностного зарядов, допускает редукцию исходных уравнений в частных производных к обыкновенным дифференциальным уравнениям для углового распределения потенциала электрического поля за счет исключения переменной $R$. В итоге удается построить следующее точное аналитическое решение уравнений $(1)-(8)$ :

$$
\begin{gathered}
\Phi_{1}=C P_{1 / 2}(\cos \theta) R^{1 / 2}, \quad \Phi_{2}=A R^{1 / 2}, \\
\rho=B R^{-3 / 2}, \quad \sigma=D R^{-1 / 2},
\end{gathered}
$$

где $A, B, C, D-$ постоянные, связанные соотношениями

$$
\begin{gathered}
C=-(-2 \alpha \operatorname{ctg} \Theta)^{1 / 2}\left[\varepsilon_{0} \sin ^{2} \Theta P_{1 / 2}^{\prime 2}(\cos \Theta)\right. \\
\left.+\varepsilon_{0}(\varepsilon-1) P_{1 / 2}^{2}(\cos \Theta) / 4\right]^{-1 / 2}, \\
A=C P_{1 / 2}(\cos \Theta), \quad B=-3 \varepsilon_{0} \varepsilon C P_{1 / 2}(\cos \Theta) / 4, \\
D=-\varepsilon_{0} C P_{1 / 2}^{\prime}(\cos \Theta) \sin \Theta .
\end{gathered}
$$

Здесь $P_{1 / 2}-$ функция Лежандра порядка $1 / 2, P_{1 / 2}^{\prime}-$ ее производная по аргументу.

Из условия (2), согласно которому поверхностный заряд полностью экранирует нормальную компоненту поля в жидкости, понятно, что полученные решения соответствуют режиму ограничения поверхностного тока зарядом: при увеличении тока направление поля в жидкости изменится на противоположное по сравнению с внешним. Остается вопрос, какой режим соответствует протеканию по жидкости объемного тока. В работе [20] приведено точное решение для сферически-симметричного распределения электрического поля в режиме ограничения тока объемным зарядом применительно к коронному разряду:

$$
E \propto R^{-2}\left(R^{3}-R_{0}^{3}\right)^{1 / 2}
$$

где $R_{0}$ - радиус активного электрода, на котором поле обращается в нуль (это решение формально 


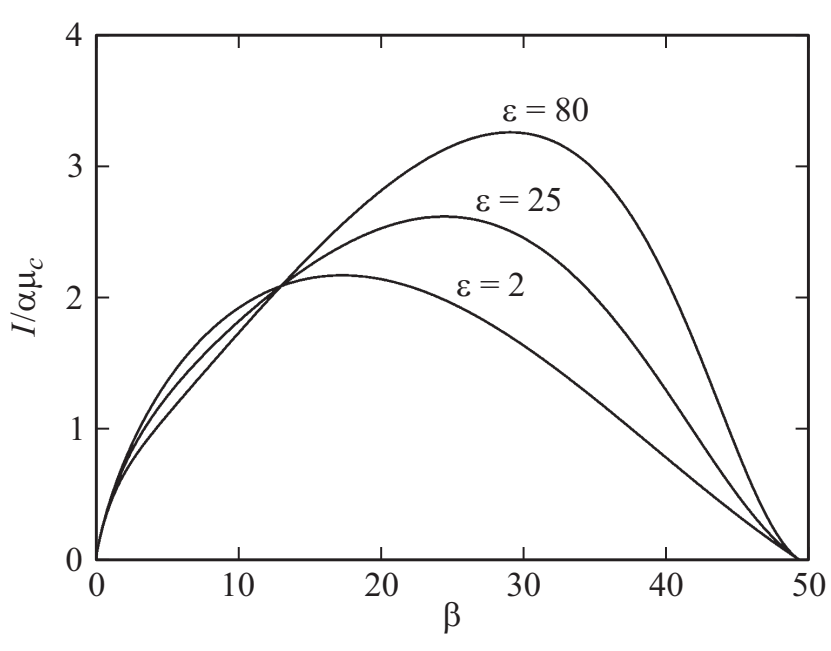

Зависимость протекающего через конус полного тока $I$ (нормированного на величину $\alpha \mu_{c}$ ) от угла полураствора конуса $\beta$ при $\mu_{a}=\mu_{c}$ и $\varepsilon=2,25,80$.

соответствует системе электродов, представляющих собой концентрические сферы). Данная зависимость переходит в используемую нами $\left(E \propto R^{-1 / 2}\right)$ при $R_{0} \rightarrow 0$. Таким образом, решение (9), несмотря на обращение в бесконечность напряженности поля в начале координат, соответствует режиму ограничения тока объемным зарядом.

Полученное точное решение (9) задачи (1)-(8) позволяет найти величину электрического тока, протекающего через конус в режиме ограничения электрическим зарядом:

$$
\begin{gathered}
I=I_{c}+I_{a}, \quad I_{c}=\pi \mu_{c} D A \sin \beta, \\
I_{a}=-\pi \mu_{a} A B(1-\cos \beta),
\end{gathered}
$$

где $I_{c}$ - ток катионов по поверхности конуса, а $I_{a}$ - ток анионов в его объеме. Типичные зависимости полного тока от угла раствора конуса для различных проницаемостей жидкости при $\mu_{a}=\mu_{c}$ показаны на рисунке. Они являются немонотонными. Ток обращается в нуль при $\beta=0$ и $\beta_{T}$; соответственно, он достигает максимума между указанными значениями. Как видно из рисунка, максимум сдвигается в сторону больших углов раствора с ростом проницаемости $\varepsilon$.

В заключение отметим, что характер зависимостей, основанных на найденных нами точных решениях, отличается от построенных в работах $[16,17]$ с использованием „slender body approximation“. Если для точных решений ток всегда ограничен, то для приближенных решений из $[16,17]$, полученных для предела малых углов $\beta$ и больших проницаемостей $\varepsilon$, ток обращался в бесконечность при $\beta \approx \sqrt{8 / \varepsilon \ln \varepsilon}$.

Работа выполнена при частичной поддержке Российского фонда фундаментальных исследований (проекты № 17-08-00430, 18-38-00313, 19-08-00098) и УрО РАН (проект № 18-2-2-15).

\section{Список литературы}

[1] Tonks L. // Phys. Rev. 1935. V. 48. P. 562-568.

[2] Френкель Я.И. // ЖЭТФ. 1936. Т. 6. В. 4. С. 347-350.

[3] Melcher J.R. Field-coupled surface waves. Cambridge: MIT Press, 1963. 190 p.

[4] Zubarev N.M. // Physica D. 2001. V. 152-153. P. 787-793.

[5] Zubarev N.M. // Phys. Fluids. 2006. V. 18. P. 028103.

[6] Кочурин Е.А. // Письма в ЖТФ. 2019. Т. 45. В. 3. С. 7-9.

[7] Kochurin E.A., Zubarev N.M., Zubareva O.V. // Phys. Rev. E. 2013. V. 88. P. 023014.

[8] Габович М.Д. // УФН. 1983. Т. 140. В. 1. С. 137-151.

[9] Boltachev G.Sh., Zubarev N.M., Zubareva O.V. // Phys. Rev. E. 2008. V. 77. P. 056607

[10] Жакин А.И. // УФН. 2013. Т. 183. В. 2. С. 153-177.

[11] Taylor G.L. // Proc. Roy. Soc. Lond. A. 1964. V. 280. P. $383-$ 397.

[12] Ramos A., Castellanos A. // Phys. Lett. A. 1994. V. 184. P. 268-272.

[13] Li H., Halsey T.C., Lobkovsky A. // Europhys. Lett. 1994. V. 27. P. $575-580$.

[14] Зубарев Н.М. // Письма в ЖЭТФ. 2001. Т. 73. В. 10. С. 613 617.

[15] Zubarev N.M. // Phys. Rev. E. 2002. V. 65. P. 055301.

[16] Subbotin A.V., Semenov A.N. // JETP Lett. 2018. V. 107. P. 186-191.

[17] Subbotin A.V., Semenov A.N. // Phys. Fluids. 2018. V. 30. P. 022108.

[18] De La Mora J.F. // J. Fluid Mech. 1992. V. 243. P. 561-574.

[19] Субботин А.В. // Письма в ЖЭТФ. 2014. Т. 100. С. 741746.

[20] Sigmond R.S.// J. Appl. Phys. 1982. V. 53. P. 891-898. 